\title{
Beitrag zur Prognose der akuten und subakuten Encephalitis und Poliencephalomyelitis ${ }^{1}$ ).
}

\author{
Von \\ Heinrich Higier (Warschau).
}

(Eingegangen am 30. Januar 1910.)

Oppenheims Verdienst ist es unbedingt, auf die relativ günstige Prognose mancher schwerer akuter Hirnleiden, speziell der nicht eitrigen Encephalitis, schon vor Jahren in seiner Monographie über diesen Gegenstand im Nothnagelschen Handbuch der Pathologie und Therapie hingewiesen zu haben. Nonnes Arbeiten über die heilbaren Pseudotumores cerebri haben uns auf dem Gebiete der Prognose einen Schritt vorwärts gebracht. Inzwischen sind eine Reihe weiterer beachtenswerter Mitteilungen zu diesen Fragen erschienen (Hochhaus, Finkelnburg, Eschba u m), in denen die reichen Erfahrungen einzelner Autoren noch einmal kritisch zusammengestellt worden sind.

Gegen das beigebrachte Material, auf das sich die Schlußfolgerungen hinsichtlich der. Prognose stützen, wird immer der Einwand erhoben werden können: die Diagnose der Encephalitis, Poliencephalomyelitis und verwandter Umstände besitze noch keine so feste Grundlagen, daß sie bei fehlender Autopsie über jeden Zweifel erhaben sei. Da hilft aber nichts. Man muß nur diesem Einwand dadurch vorzubeugen suchen, daß man, wie Oppenheim sagt, die Diagnose so gut begründet, wie es nach den zu Gebote stehenden Kenntnissen möglich ist. Die neurologische Diagnose - speziell eine cerebrospinale - wird immer bei einem günstig abgelaufenen Falle eine Wahrscheinlichkeitsdiagnose bleiben. Ủberzeugend können selbstverständlich nur solche Fälle sein, bei denen der Patient eine längere Zeit nach der ausgeheilten Encephalitis an einer zufälligen Krankheit stirbt und seziert wird. Da es sich in meinen zu demonstrierenden Fällen meist um solche handelt, deren günstiger Ausgang 2-3 Jahre hindurch verifiziert wurde und deren Beginn somit in das Jahr 1906--1907 zurückreicht, so dürfte es nicht auffallen, daß bei sämtlichen die Wassermannsche Reaktion nicht ausgeführt wurde und daß bei manchen auch der Befund der Lumbal-

1) Nach drei in der Neurologischen Sektion der Warschauer Arztegesellschaft im Jahre 1907-1909 stattgefundenen Demonstrationen. 
punktion ausgeblieben ist. Ich will bei der Demonstration nur das Wichtigste aus den Krankengeschichten anführen, da uns doch im folgenden hauptsächlich der Verlauf und der Ausgang interessieren sollen.

\section{, Beobachtung 1.}

Bei einem 7 monatlichen, normal geborenen, von gesunden Eltern abstammenden Kinde entwickelte sich am 28. IV. 07 bei wenig gesteigerter Temperatur $\left(38,2^{\circ}\right)$ binnen eines Tages eine Lähmung im Bereiche mancher Hirnnerven.

Bei der Untersuchung lassen sich jetzt noch, am Schluß der dritten Woche, feststellen ${ }^{1}$ ): auffallende doppelseitige Ptose, besonders linksseitige, Lähmung des linken Rectus internus, Strabismus externus, gut erhaltene Pupillenreaktion, Parese des rechten Facialis in seinen sämtlichen drei Zweigen mit Herabsetzung der elektrischen Erregbarkeit daselbst für galvanische und faradische Stromesarten.

Der Zustand besserte sich mit jedem Tag, als ganz unerwartet am 29. Tage der Krankheit unter schweren Allgemeinerscheinungen und hoher Temperatursteigerung eine Lähmung der linken Gesichtshälfte und Herpes zoster facio-cervicoauricularis an derselben Seite sich einstellte. Am Nervensystem ließ sich sonst nichts Pathologisches feststellen außer einer schlaffen Parese des rechten Beines mit Fehlen des Patellarreflexes.

Es ist nun nach etwa $21 / 2$ Jahren keine Spur nachgeblieben von der Diplegia facialis und vom Schielen. Was besonders in die Augen fällt, ist die persistierende partielle Lidsenkung beiderseits und eine wenig ausgesprochene Atrophie der rechten Beinmuskulatur.

Der Verlauf der Krankheit läßt sich in kurzer Zusammenfassung folgendermaßen darstellen. Ein 7 monatliches Kind erkrankt unter Fiebererscheinungen an rechtsseitiger Gesichtslähmung, linksseitiger Internuslähmung und doppelseitiger Ptose. Der Zustand bessert sich allmählich, um plötzlich nach einem Monate einem neuen Aufflackern des Krankheitsprozesses Platz zu machen, das sich in einer Parese des rechten Beines, einer linksseitigen Facialislähmung und Herpes zoster daselbst kundgibt.

Der Beginn und Verlauf lassen ohne weiteres eine Meningitis oder Rindenencephalitis ausschließen, die anamnestischen Daten und die genaue Inspektion des Kindes machen eine $\mathrm{Hirnlues}$ unwahrwahrscheinlich. Zusammenhang mit einer vorausgegangenen bestimmten Infektionskrankheit läßt sich nicht nachweisen.

Die einzige Diagnose, die hier noch am meisten plausibel erscheint, ist: entzindliche Affektion des Mittelhirns und Hinterhirns, eine der Poliomyelitis analoge Poliencephalitis. Die akute Poliencephalitis lokalisiert sich hier in den vorderen, teilweise auch hinteren Vierhügeln in der grauen Substanz, die den Aquaeductus Sylvii umgibt und hinterwärts in der Rautengrube, die den Boden des vierten Ventrikels bildet, an der Grenze zwischen Brücke und Oblongata.

1. Das Intaktbleiben der Pupillenreaktion bei doppelseitiger Ptose spricht für das Erhaltenbleiben der vordersten Oculomotoriuskerne, der Edinger-Westphalschen Kernsäule.

1) Demonstriert am 18. V. 07 . 
2. Die besonders starke Affektion der Augenmuskulatur links spricht für die intensivere Läsion der linken Vierhügel.

3. Die Parese der Gesichtsmuskeln läßt die hintere Grenze des Entzündungsherdes in der Varolschen Brücke lokalisieren.

Was diese Poliencephalitis besonders auszeichnet, sind folgende Punkte:

1. Der Beginn, das Zustandsbild und der Ausgang entsprechen bedeutend mehr dem der alltäglichen Poliomyelitis, als der eigentümlichen, von Wernicke, Thomsen u. a. beschriebenen, meist letal verlaufenden Encephalitis dieser Gegend bei Influenza, Alkoholismus und schweren Intoxikationen.

2. Ihr Verlauf ist insofern eigenartig, als nach einer einmonatlichen Remission ein neues fieberhaftes Aufflackern des infektiösen, entzündlichen Herdes stattfand, das sich in der Parese des rechten Beines und der Lähmung der linken Gesichtshälfte äußerte und das typische Bild der Poliencephalitis superior zum selteneren, nicht alltäglichen Bilde der disseminierten Poliencephalomyelitis umgestaltete. Dieser schubweise Entwickelungsgang ist in der Regel eine Eigentümlichkeit der subakuten Poliomyelitis der Erwachsenen, bedeutend seltener der akuten Poliomyelitis der Kinder.

3. Unsere ,cerebrale Poliomyelitis“, wenn man sie so nennen darf, lokalisierte sich nicht bloß loco classico in der vorderen motorischen grauen Substanz des Lumbalmarks und in den Kernen der Augenund Gesichtsmuskulatur, sondern auch in der hinteren grauen Substanz, der sensiblen, die der Trigeminusbahn entspricht, wofür der schmerzhafte Herpes zoster spricht, der bekanntlich nach den neuesten Untersuchungen Campbells und Heads auf eine Poliomyelitis posterior hinweist.

4. Interessant ist weiterhin die Tatsache, daß nun nach $2 \frac{1}{2}$ Jahren, wo sämtliche Symptome außer der doppelseitigen Ptose geschwunden sind, unser Fall beim ersten Anblick ganz den Eindruck einer kongenitalen Ptose macht aus dem Gebiete des Moebiusschen infantilen Kernschwundes. Sollten nach Jahren die anamnestischen Daten des Säuglingsalters verloren gehen oder mißtrauisch beurteilt werden, so wird die Ptose unbedingt von den meisten Ärzten zur eben genannten kongenitalen Gruppe gezählt werden. Und tatsächlich gehört wahrscheinlich ein nicht geringer Teil der sog. angeborenen Ptosen oder des angeborenen Schielens zur Kategorie der in der frühesten Kindheit ev. in utero durchgemachten entzündlichen infektiösen Poliencephalitis.

5. Der letzte Punkt, auf den ich besonders hinweisen möchte und der unten noch eingehender besprochen werden soll, ist der ganz günstige Ausgang des relativ schweren Krankheitsfalles von Poliencephalomyelitis acuta recidivans. 


\section{Beobachtung 2.}

Das 17 jährige Mädchen, immer gesund und aus einer gesunden Familie abstammend, blühend aussehend, fing an im Oktober 1906 bei normaler Temperatur über Kriebeln zu klagen in der linken Hand und im rechten Fuß. Im Laufe des nächsten Monats stellten sich starke Kopfschmerzen mit Erbrechen ein, zu denen sich allmählich eine rechtsseitige Hemiparese, speziell des Beines, hinzugesellte, Lähmung der rechten Hand und Abnahme der Empfindlichkeit an der linken Körperhälfte.

Dreimal wiederholten sich epileptiforme Krämpfe mit komplettem Bewußtseinsverlust, mit Bevorzugung der rechten Gesichtsmuskulatur und nachfolgender Amnesie.

Im Laufe der nächsten Wochen bei Abwesenheit von Nackenstarre nahmen die Kopfschmerzen ab, das Erbrechen hörte auf, dagegen ließ sich feststellen: links Hemiparese, spastische Erscheinungen in sämtlichen Extremitäten, Intentionszittern und starke Ataxie der Arme und Hände. Am Schluß des dritten Monats war eine schwach ausgesprochene Neuritis optica an beiden Augen aufgetreten.

Seit Anfang März 1907 bessert sich der Zustand der Kranken langsam ohne Remissionen fortwährend, so daß sie zurzeit1) nur über Prickeln in der linken Hand und Ameisenlaufen im rechten Fuß klagt.

Objektiv läßt sich feststellen: schwach spastischer Gang, rechts leichte Hemiparese mit Einschluß der Gesichtsmuskulatur, Hyperextension der rechten Zehe mit dem Babinskischen Phänomen, Lebhaftigkeit der Sehnenreflexe, leichtes Zittern der Hände, normales Verhalten der Bauchreflexe, kein Nystagmus, kein Skandieren, Abschwächung des Tast- und Schmerzgefühls und insbesondere des Muskelsinnes in der linken Hand und dem rechten Fuß.

Nach 3 Jahren sind nur noch die eben genannten subjektiven und objektiven Sensibilitätsstörungen nachgeblieben.

Resumé: Es handelt sich um ein gesundes 17 jähriges Fräulein, bei dem sich im Laufe dreier Monate langsam das Bild eines schweren fieberlosen Hirnleidens entwickelte: Kopfschmerzen, Erbrechen, spastische Diparese, epileptiforme Anfälle, linksseitige Lähmung, Intentionszittern, Ataxie, Sensibilitätsstörungen in der linken Hand und im rechten FuB, bilaterale Neuritis optica. Bis auf die Sensibilitätsaffektion hat sich alles spurlos zurückgebildet.

Der Krankheitsverlauf machte im ersten Stadium den Eindruck einer schleichend sich entwickelnden Meningitis, im zweiten einer rasch wachsenden Neubildung tuberkulöser oder syphilitischer Natur. Eine energische Jod- und Quecksilberkur blieb in den ersten Monaten der Erkrankung ganz ohne Effekt. Für Lues und Tuberkulose sprach auch absolut nichts, weder bei der sonstigen Untersuchung der Kranken und der nächsten Familienangehörigen noch bei der skrupulösesten Aufnahme der anamnestischen Daten. Von sämtlichen Diagnosen, an die ich im Laufe der Beobachtung der Patientin dachte (akquirierte Hydrocephalie, circumscripte tuberkulöse Meningitis, Pseudotumor, akute Herdsklerose, diffuse subakute Encephalitis) muß ich nun nach Ablauf dreier Jahre mich mit großer Wahrscheinlichkeit für letztere aussprechen, für diejenige schleichend sich entwickelnde fieberlose Encephalitis, von der Oppenheim mit Recht

I) Demonstriert am 18. V. 07 und 20. VI. 08. 
sich ausdrückt, daß sie in den ersten Stadien von beginnender Geschwulat absolut nicht $\mathrm{zu}$ unterscheiden ist.

Auf die nähere Lokalisation gehe ich hier nicht ein. Es sei nur so viel gesagt, daß die zwei vermutlichen Entzündungsherde wahrscheinlich in der weißen Substanz der Brücke liegen, in verschiedener Höhe derselben und ohne miteinander in der Mittellinie zu konfluieren: der rechte melir dorsomedial, der linke ventrolateral. Affiziert ist von denselben hauptsächlich die Schleife und das Haubenfeld, in schwächerem Grade die Pyramidenbahn, verschont die naheliegende supranucleäre bulbäre Bahn und das hintere Längsbündel.

Von einer sich aus der Encephalitis entwickelnden Herdsklerose ist vorläufig keine Rede, zumal ich. mit der akuten Form der multiplen Sklerose und ihrer Stellung zur disseminierten Encephalomyelitis bekannt, wiederholt vergebens Symptome der ersteren gesucht habe. Es fehlt zwar an sicheren Kriterien, um im akuten Stadium entscheiden zu können, ob die Heilung eine dauernde und vollständige sein wird. In unserem Falle glaube ich jedoch 3 Jahre nach dem Beginn der Krankheit die Prognose in dem ziemlich schweren Falle von subakuter disseminierter Encephalitis recht günstig stellen zu können und von einer beinahe kompletten Heilung sprechen zu dürfen.

\section{Beobachtung 3.}

Der 17 jährige Knabe, auf Lues und Potus nicht verdächtig, bekam vor $13 / 4$ Jahren etwa 24 Stunden nach anstrengender physischer Arbeit Kopfschmerzen, Hitze und Erbrechen. Im Laufe weniger Tage entwickelten sich bei gesteigerter Temperatur Lähmung der Beine und Blasendarmstörungen. Keine Schmerzen und keine Nackenstarre waren vorhanden, dagegen störten sehr intensiv den Patienten Doppeltsehen und Ataxie der linken Körperhälfte. Die Störungen seitens der Blase und des Darmes, bestehend in Retenz und Inkontinenz, schwanden am Schluß der zweiten Woche spurlos.

Es fehlen zurzeit1), wie auch während der ganzen Krankheitsdauer: Intelligenz-, Bulbär- und Sensibilitätsstörungen. An den Augenmuskeln und der Gesichtsmuskulatur ist nichts vorhanden außer einer ausgesprochenen Abducensparese links. An den unteren Extremitäten atrophische Parese sämtlicher Fußmuskeln des linken Beines und des Peroneusgebietes am rechten Beine, Schwäche der Patellarreflexe beiderseits, Fehlen der Achillessehnenreflexe und der Hautreflexe seitens der Bauchmuskulatur und der Cremasteren.

Enorm ausgesprochene Lendenlordose. Das von dem am weitesten vorspringenden Brustwirbel im Stehen heruntergelassene Lot fällt auf die Mitte des Kreuzbeins. Die Lordose ist stark beim Stehen und Liegen, schwindet beim Sitzen, steigert sich im Liegen beim Versuch die Beine aufzuheben. Unmöglichkeit des sich Aufrichtens von der liegenden Position resp. des gleichzeitigen Erhebens beider Oberschenkel. Man fühlt bei diesem Versuch eine äußerst geringe Anspannung der Recti, die als ganz schmale Stränge von $1 / 3$ der normalen Breite zu fühlen sind. Von den schrägen und queren Bauchmuskeln sind nur die unteren Hälften beim Preßversuch deutlich kontrahiert zu fühlen.

1) Demonstriert am 20. VI. 08. 
Die Mm. sacrolumbales sind normal elektrisch erregbar, die Mm. obliqui und transversi adbominis sind schwach erregbar, die Recti ganz unerregbar. Das Hervorbringen niedriger Töne ist stark alteriert, das Husten, Niesen, Atmen nicht beeinträchtigt.

'Auf Grund der genannten Erscheinungen läßt sich die Krankheitsgeschichte folgendermaßen zusammenfassen: bei einem 17 jährigen Jüngling ent wickelt sich unter Temperatursteigerung ziemlich akut binnen wenigen Tagen ein Bild, in dem neben Gehirnerscheinungen (Kopfschmerzen, Erbrechen, linksseitige Ataxie, Abducenslähmung) solche seitens des Rückenmarks (Paraplegia inferior mit Muskelschwund, Bauchmuskelparesen, Blasenmastdarmstörungen, Verlust der Sehnen- und Hautreflexe, Wirbelsäulendeformation) prävalieren.

Es ist nicht schwer zu beweisen, daß es sich hier bei Abwesenheit bestimmter ätiologischer Momente nur um eine diffuse akute genuine Encephalomyelitis gehandelt hat. Bezeichnend war von vornherein für die Encephalitis gegenüber den Intoxikationszuständen, daß frühzeitig Herdsymptome zur Entwicklung kamen und die Symptomatologie beherrschten.

Das Eigentümliche des Falles liegt zunächst im Alter des Patienten (Poliencephalomyelitis adultorum) und dann in der anatomischen Lokalisation der Krankheitserscheinungen, die sowohl die Hirnrinde und das Mittelhirn als die graue Substanz des Dorsal- und Lumbalsakralmarks einnimmt. Die Affektion der Bauchmuskulatur und des Cremasters, eines anatomischen Derivats der Bauchmuskeln, erklärt uns genügend den Verlust der Bauch- und der Cremasterreflexe. Die über zwei Wochen anhaltende Lähmung der Blasen- und Darmfunktionen gehört zu den seltensten Ausnahmen bei akuter Poliomyelitis und weist auf einen rasch zurückgebildeten Entzündungsherd in der grauen Substanz auf der Höhe des 3. und 4. Sakralsegmentes hin.

$\mathrm{Zu}$ den seltenen Symptomen gehört auch die partielle Lähmung der Bauchmuskulatur mit den Abweichungen der elektrischen Erregbarkeit. Aus der relativ gut erhaltenen Erregbarkeit auf elektrische Reize mancher Muskeln (sakrolumbalis, transversi, obliqui) bei fehlender Reaktion in anderen Bauchmuskeln (recti) darf keineswegs der Schluß gezogen werden, daß die motorischen Kerne für die Recti höher gelagert seien als die für die Obliqui, da es sich hier beim Intaktbleiben der Oberschenkelmuskulatur um zwei voneinander getrennte Rückenmarksherde zu handeln scheint, von denen einer die Bauchmuskulatur, der andere die Unterschenkel- und Fußmuskulatur alteriert hat. Daß der Ausfall der Bauchpresse das Seinige zur Entstehung der Blasenmastdarmretention beigetragen hat, darf nicht geleugnet werden, wenngleich die Hauptrolle, wie schon oben erwähnt, der Affektion der vesicorektalen Centren zukommt, speziell sofern die Inkontinenz in Betracht gezogen wird.

Auf Grund des Verhaltens der Lordose beim Sitzen, Stehen und Liegen unseres Patienten würde sich die landläufige Ansicht $D$ uch e n nes 
über den Mechanismus der Entstehung derartiger Wirbelsäuledeformøtionen nicht aufrecht erhalten lassen.

Besonders interessant war für mich der demonstrierte Fall hauptsächlich durch seinen besonders günstigen Ausgang trotz der umfangreichen entzündlichen Herde, die er im cerebrospinalen System hinterlassen hat.

\section{Beobachtung 4.}

Der 12jährige Arbeitergehilfe, stets gesund und ex stirpe bona stammend, bekam vor 10 Monaten einen schweren Hieb in die Scheitelgegend. Die nachgebliebene Blutunterlaufung und Hautschwellung schwand nach einer Woche spurlos. Etwa I0 Wochen darauf entwickelten sich langsam und fieberlos die ersten Kopfschmerzen mit Erbrechen.

Binnen kurzer Zeit lag das klassische Bild einer schweren Hirnkrankheit vor mit ausgesprochenen allgemeinen Druck- und Herderscheinungen: Arhytmie des Pulses, Cephalalgie, fortwährendes Aufstoßen und Erbrechen, Zittern der Zunge, nasale, verwaschene explosive Sprache, links. seitige Hemiparese, Ataxie des $R$ umpes und der oberen und unteren Extremitäten im Stehen, Sitzen und Liegen, gesteigerte Sehnen. reflexe, doppelseitiger Clonus pedis, bedeutende Einengung des Gesichtsfeldes auf Wei $\beta$ und Farben, beiderseitige Staungspapille und herabgesetzte Sehschärfe (rechts auf $1 / 3$, links a uf $1 / 2$ ). Der schwere Zustand dauerte über 4 Monate.

Seit etwa 3 Monaten fühlt sich der Knabe ziemlich gut. Man findet zurzeit1) bei dem blühend aussehenden Patienten auch bei genauester Untersuchung nichts mehr als eine Stauungspapille, deutlicher ausgesprochen rechts, ohne bedeutende Herabsetzung der Sehschärfe.

Ich will hier nicht länger aufhalten mit dem Gedankengang, der uns im Laufe der Krankheitsentwicklung leitete. Bei exspektativer Behandlung ließen wir nach wenigen Wochen die meisten in Betracht kommenden und $\mathbf{z u}$ ev. chirurgischen Einschreiten auffordernden Diagnosen fallen: Schädelknochenbruch mit Impression, pachymeningeales Hämatom, Spätapoplexie, GehirnabsceB, circumscripte Meningitis serosa.

Liegt hier definitive Heilung vor oder eine gewöhnliche Remission? Es ist hier geradezu schwer, von einer Remission s. s. zu sprechen, wo von schwerster Ataxie, Erbrechen, Kopfschmerzen, Bulbärsymptomen und sonstigen Herd- und Allgemeinerscheinungen seit 3 Monaten keine Spur nachgeblieben ist und der Patient seine frühere anstrengende Beschäftigung als Arbeitergehilfe wieder aufgenommen hat. Ich unternehme es nicht, endgültig die Frage zu entscheiden, ob hier ein Pse udot u mor im Sinne Non nes oder eine akquiererte $\mathrm{H}$ ydrocephalie mit oder ohne Tumor vorliegt. Die weitere genaue Beobachtung soll das entscheiden.

Zwei Punkte mahnen uns zur Vorsicht bei der Diagnose- und Prognosestellung: das vorausgegangene Trauma und die trotz kompletter

1) Demonstriert am 24. VII. 09. 
Restitution stehen gebliebene Stauungspapille. Mir persönlich imponiert der Fall als eigentümlich verlaufende, spät aufgetretene, in Heilung begriffene traumatische Meningoencephalitis oder Meningitis serosa.

Ich will nur aufmerksam machen, daß hie und da in Ausnahmefällen ko m plette Remissionen auch bei Tumoren ganz lange anhalten. Einen Fall kenne ich, wo das beinahe ein Jahr scheinbar geheilte Kleinhirnsarkom plötzlich rezidivierte und schnell zum Exitus führte.

\section{Beobachtung 5 .}

Das 20 jährige, stark anämische, von tuberkulösen Eltern abstammende Mädchen bekam am 13. IV. 08 plötzlich ohne Prodrome unter hoher Temperatur und bei schwerem Allgemeinbefinden typische epileptische Anfälle mit absolutem Bewußtseinsverlust, die am nächsten Tage einem sohweren Status hemiepilepticus Platz machten.

Am 3. Tage hörten die Anfälle auf, die Kranke blieb jedoch ganz bewußtlos, die Temperatur bielt sich bei $39,5^{\circ}$, die Atmung war von Cheyne-Stokesschem Typus, die Respiration beschleunigt, die Pupillen stark erweitert und sämtliche Sehnenreflexe pathologisch gesteigert. Nach 4 Tagen kehrte das Bewußtsein allmählich zurück, und es ließ sich feststellen neben Kopfschmerzen, Erbrechen und Harnretention eine komplette rechtsseitige Hemiplegie mit motorischer Aphasie. Die Harnretention schwand nach einer Woche, die Sprache kehrte langsam am Schluß der zweiten Woche zurück und blieb eine Zeitlang ziemlich unverständlich.

Die Temperatur schwankte ca. 15 Tage zwischen 37,2 und $39^{\circ}$. Krämpfe wiederholten sich nicht mehr.

Die Harnanalyse ergab normale Verhältnisse, bei der ophthalmoskopischen Inspektion hat sich nichts Nennenswertes eruieren lassen.

Die Lumbalpunktion am fünften Krankheitstage ergab keinen erhöhten Druck, keine Mikroorganismen, vereinzelte kleine Lymphoscyten und mehrkernige neutrophile Zellen, $1 / 2 \%$ Eiweiß.

Der Zustand besserte sich progressiv, so daß das Fräulein zurzeit ${ }^{1}$ ) gar nicht schlecht geht mit einer Spur von Parese des rechten Beines, mit Clonus pedis und Babinskischem Zehenphänomen; am Arm ist eine ausgesprochene spastische Monoplegie ohne Apraxie vorhanden und am Gesichte eine ziemlich deutliche Parese sämtlicher Facialisäste. Muskelatrophien sind nicht vorhanden. Die Sprache ist langsam, explosiv, skandierend und saccadiert. Von der motorischen Aphasie ist keine Spur nachgeblieben. In der sensiblen Sphäre, in den Pupillen, Hirnnerven und in der Sphäre der Intelligenz ist nichts Pathologisches zu finden. Innere Organe gesund.

Es handelte sich somit um ein 20 jähriges, aus tuberkulöser Familie stammendes anämisches Fräulein, das plötzlich unter schweren Allgemeinerscheinungen und rasch sich steigernder Tem. peratur das BewuBtsein verlor und schwere epileptische Anfälle bekam, die mehrere Tage anhielten. Nach Zurückkehr des Bewußtseins a $\mathrm{m}$ vierten Krankheitstage lie $B$ sich eine Hemiplegie mit motorischer Aphasie feststellen, die allmählioh abnahm, so daß in der neunten Woche nur noch eine facio-brachiale Monoplegie mit einer eigen. tümlichen, nicht aphatischen Sprachstörung festzustellen war, bei der das Langsame, Skandierende neben dem Explosiven auffiel.

1) Demonstriert am 20. VI. 08. 
Mußte man in den ersten Tagen bei fehlender Anamnese der bewußtlosen Patientin an Urämie, Status epilepticus und an chlorotischer Sin usthrombose denken, so ließ sich schon am vierten Tage nach Rückkehr des Bewußtseins mit großer Wahrscheinlichkeit ein infektiöses Hirnleiden diagnostizieren. Es konnte bei fehlenden Eiterherden am Körper ohne weiteres ein Hir nabsce B, bei abwesendem Opistothonus und bei negativem Ergebnisse der Lumbalpunktion eine seröse oder tuberkulöse Meningitis ausgeschlossen werden.

Das klinische Bild, der Verlauf und der Ausgang sprechen für eine gutartige, nicht eitrige Encephalitis, die im Jünglingsalter gerade anämische Individuen zu bevorzugen pflegt. Nicht ganz auszuschließen war während des Fieberstadiums die sog. Meningitie en plâques der französischen Autoren, die Meningoencephalitis acuta tuberculosa circumscripta, die meist im Kindesalter auftritt und gelegentlich ganz günstig verläuft.

Daß wir in unserem Falle diejenige schwere Folgeerscheinung nicht finden, der wir bei der akuten Encephalitis der Kinder begegnen, wie schwere spastische Kontrakturen, trophische Erscheinungen am Muskelund Knochensystem, athetotische Reizerscheinungen, erklärt uns leicht das Alter der Patientin. Der plötzliche, foudroyante, epileptiforme Ausbruch der Krankheit spricht dafür, daß es neben der akuten und subakuten Form, die ich hier wiederholt zu demonstrieren Gelegenheit hatte, eine Encephalitis acutissima gibt mit ganz guter Prognose. Ich will Sie hier nicht weiter mit der Lokalisation beschäftigen : für wahrscheinlich halte ich den subkortikalen Sitz des Entzündungsherdes, der gleichzeitig die motorische Bahn für die Extremitäten neben der mimischen und phonatorisch-artikulatorischen Muskulatur affiziert hat.

Das Punctum saliens der Beobachtung sehe ich darin, daß trotz des äußerst schweren Verlaufes des akuten Hirnleidens im Laufe der ersten Woche der Ausgang dennoch sich ziemlich günstig für die junge Patientin gestaltet hat.

Ich bin am Schluß meiner Demonstrationen. Ich führte eine Reihe von schweren Fällen vor, die teils cerebraler, teils cerebrospinaler Natur sind, die sämtlich einen akuten oder subakuten Verlauf nahmen und unerwarteterweise durch ihren sehr günstigen Verlauf sich auszeichneten. Nirgends versuchte ich die strenge Trennung durchzuführen in die üblichen drei Hauptformen der Encephalitis, die Wernickesche, die Strümpell-Leichtensternsche und die gemischte, da sie sich klinisch nicht selten miteinander verbinden und, wie Sie sahen, derart durch Rückenmarkserscheinungen kompliziert werden, daß zuweilen der Heine-Medinsche spinale Symptomenkomplex ganz in Vordergrund des Krankheitsbildes tritt. $\mathrm{Zu}$ beachten bleibt nur, daß die Prognose auch in denjenigen Fällen günstig sich gestalten kann, wo die Hirn- 
symptome, encephalitische und poliencephalitische, die ganze Zeit im Zustandsbilde prävalieren.

Auf das therapeutische Eingreifen den günstigen Ausgang zurückzuführen, liegt kein Grund vor. Die Therapie war in der Mehrzahl unserer Fälle exspektativ und indifferent mit Ausnahme des einen, wo eine diagnostische Lumbalpunktion ausgeführt, und des anderen, wo eine Quecksilberbehandlung eingeleitet wurde, in beiden Fällen jedoch ohne unmittelbaren deutlichen Effekt. 